\title{
Vitamin D Deficiency Is Associated With Impaired Muscle Strength And Physical Performance In Community-Dwelling Older Adults: Findings From The English Longitudinal Study Of Ageing
}

This article was published in the following Dove Press journal:

Clinical Interventions in Aging

\author{
Niamh Aspell' \\ Eamon Laird (iD) ${ }^{\prime}$ \\ Martin Healy ${ }^{2}$ \\ Brain Lawlor ${ }^{3}$ \\ Maria O'Sullivan' \\ 'Department of Clinical Medicine, School \\ of Medicine, Trinity College Dublin, \\ Trinity Centre for Health Sciences, \\ Dublin 8, Ireland; ${ }^{2}$ Department of \\ Biochemistry, St James's Healthcare \\ Campus, Dublin 8, Ireland; ${ }^{3}$ Department \\ of Medical Gerontology and Institute of \\ Neurosciences, Trinity College, Dublin, \\ Ireland
}

\begin{abstract}
Purpose: Maintaining skeletal muscle function throughout life is a crucial component of successful ageing. Adequate vitamin D status may be important in preserving muscle function. We aimed to determine the association between impaired muscle function and serum vitamin D status in community-dwelling older adults. Falls were explored as a secondary aim.

Methods: Data were analyzed from adults aged $\geq 60$ years, from Wave 6 of the English Longitudinal Study of Aging (ELSA). Handgrip strength (HGS) and the short physical performance battery (SPPB) were employed as measures of muscle strength and physical function, respectively. Serum 25-hydroxyvitamin D [25(OH)D] was assessed with concentration $<30 \mathrm{nmol} / \mathrm{L}$ classed as vitamin $\mathrm{D}$ deficient.
\end{abstract}

Results: The study comprised 4157 community-dwelling adults with a mean age of 69.8 (SD 6.9). Overall, 30.6\% had low HGS and $12.7 \%$ had low SPPB $(\leq 6)$. Participants with the lowest serum $25(\mathrm{OH}) \mathrm{D}(<30 \mathrm{nmol} / \mathrm{L})$ had the highest prevalence of impaired muscle strength and performance ( $40.4 \%$ and $25.2 \%$ ) compared with participants with levels $\geq 50 \mathrm{nmol} / \mathrm{L}(21.6 \%$ and $7.9 \%)$. Consistent with this, vitamin D deficiency $(<30 \mathrm{nmol} / \mathrm{L})$ was a significant determinant of low HGS (OR 1.44 [1.22, 1.71], p <0.001) and poor physical performance (OR 1.65 [1.31, 2.09 ] $\mathrm{p}<0.001)$ in the logistic regression models. Older adults partaking in regular moderate physical activity had significantly lower odds of impaired muscle strength (OR $0.65[0.58,0.79])$ and physical function (OR $0.30[0.24,0.38]$ ), $p<0.001$, respectively. Single or multiple falls (15.8\% and $10.5 \%$ in past year) were not associated with vitamin D status.

Conclusion: Vitamin D deficiency was associated with impaired muscle strength and performance in a large study of community-dwelling older people. It is generally accepted that vitamin $\mathrm{D}$ deficiency at the $<30 \mathrm{nmol} / \mathrm{L}$ cut-off should be reversed to prevent bone disease, a strategy that may also protect skeletal muscle function in ageing.

Keywords: vitamin D, 25-hydroxyvitamin D, ageing, muscle function, short physical performance battery

\section{Introduction}

Maintaining muscle mass and function throughout life is a crucial component of successful ageing. The progressive loss of skeletal muscle mass accompanied by a loss of muscle strength or performance, in ageing, are features of primary sarcopenia. ${ }^{1,2}$ This functional decline negatively impacts on mobility, falls risk, independence, quality of life and healthcare costs. ${ }^{2-5}$ The condition is a major
Correspondence: Maria O'Sullivan Human Nutrition, School of Medicine, Trinity College Dublin, Trinity Centre for Health Sciences, St James's Healthcare Campus, Dublin 8, Ireland

Tel +353 I 8964039

Email maria.osullivan@tcd.ie 
public health issue, estimated to effect between $6 \%$ and $22 \%$ of older adult populations ${ }^{6}$ and up to half of adults over 80 years. Resistance exercise is well evidenced in maintaining muscle function ${ }^{6}$ while nutritional factors such as adequate dietary protein, ${ }^{6-9}$ and potentially adequate vitamin D status, ${ }^{8-10}$ have shown benefit. Globally, vitamin $\mathrm{D}$ deficiency is widespread ${ }^{11}$ particularly in older populations $^{12,13}$ and optimizing vitamin $\mathrm{D}$ status may be a practical and cost-effective approach that supports skeletal muscle function.

Findings from animal models and laboratory studies show that vitamin D can improve muscle quality and strength and reduce intramuscular fat and inflammation. ${ }^{14}$ Epidemiological $^{15-17}$ and intervention studies ${ }^{8,10}$ further support associations between circulating 25-hydroxyvita$\min \mathrm{D}[25(\mathrm{OH}) \mathrm{D}]$ or vitamin $\mathrm{D}_{3}$ supplementation and muscle function in older adults, though more recent findings suggest no benefit. ${ }^{18-20}$ For falls outcomes, vitamin D supplementation appears beneficial for older people living in care homes ${ }^{21}$ or with low vitamin D status, ${ }^{22}$ but the findings are inconsistent for the wider population of community-dwelling older adults. ${ }^{22}$ Some studies, conversely, have found an increase in falls rate with large bolus vitamin D supplementation. $^{23}$

Several methodological differences are apparent across the studies that have investigated vitamin D and musculoskeletal outcomes in community-dwelling older adults; these include the population age, health and lifestyle characteristics, vitamin D status and the outcome measures employed for skeletal muscle. A key aspect is the degree to which study samples capture participants with established vitamin D deficiency or at-risk of deficiency. Such risk factors include advanced age, lower socioeconomic status, poorer health status, non-vitamin D supplement users, non-white ethnicity ${ }^{12,24}$ or mobility impairment. ${ }^{10}$ An important consideration is if older adults with vitamin $\mathrm{D}$ deficiency have impaired muscle function and, if so, would raising $25(\mathrm{OH}) \mathrm{D}$ status or reversing deficiency improve or preserve function. Skeletal muscle measures employed in studies are typically brief assessment of muscle strength or function including handgrip strength (HGS), gait speed or sit to stand tests. ${ }^{9}$ More comprehensive assessments, such as the short physical performance battery (SPPB) ${ }^{25}$ or imaging techniques, that assess lower limb muscle function or muscle quality ${ }^{2,10,16}$ are important to add value to vitamin $\mathrm{D}$ research in this field.

The present study aimed to estimate the prevalence of impaired muscle function (HGS and SPPB) and determine the association between these variables and serum vitamin D status in community-dwelling older adults from the English Longitudinal Study of Aging (ELSA). As a secondary aim, we explored the association between vitamin D status and falls.

\section{Materials And Methods Study Design And Population}

The study was based on data from community-living older people who participated in ELSA $^{26}$ Wave 6 (2012-2013) as this represented the first analysis of $25(\mathrm{OH}) \mathrm{D}$. The ELSA study was conducted in line with the Declaration of Helsinki and was approved by the London Multicentre Research Ethics Committee (MREC 01/2/91). Written informed consent was obtained from all participants (https://www.elsa-project.ac.uk/).

For the present study, the inclusion criteria were as follows: measured serum $25(\mathrm{OH}) \mathrm{D}$, adults aged $\geq 60$ years and participation in assessments of skeletal muscle function, namely HGS and components of the SPPB. Lower limb measures, such as gait speed, were assessed only in adults aged 60 years or over in ELSA. Overall, 7731 participants completed the nurse health visits, allowing the collection of biological data such as blood samples. The most common reasons for missing or unavailable blood samples were for participants who did not wish to consent to, or were considered ineligible for, blood sampling (e.g. bleeding disorders or anticoagulant use). After applying the inclusion criteria, the final analytical study sample comprised of 4157 subjects (Figure 1).

\section{Serum 25(OH)D Measurement And Deficiency}

Serum 25(OH)D was assessed from fasting blood sample and analyzed using Diasorin Liaison 25-hydroxyvitamin D immunoassay. All assays were performed in duplicate, the $\mathrm{CV}$ values ranged from $8.7 \%$ to $9.4 \%$. The laboratory responsible for analysis of vitamin D for ELSA (Royal Victoria Infirmary, Newcastle upon Tyne, UK) participated in the Vitamin D External Quality Assessment Schemes (DEQAS). All blood sampling occurred from January 2012 to July 2013. Vitamin D status was classified according to the Institute of Medicine (IOM) guidelines, ${ }^{27}$ with deficiency compatible with bone health risk defined as $25(\mathrm{OH}) \mathrm{D}<30$ $\mathrm{nmol} / \mathrm{L}$, and insufficiency as $<50 \mathrm{nmol} / \mathrm{L}^{27}$ Season was categorized using the extended vitamin $\mathrm{D}$ calendar for summer (April-Sept) and winter (Oct-March). ${ }^{28}$ 


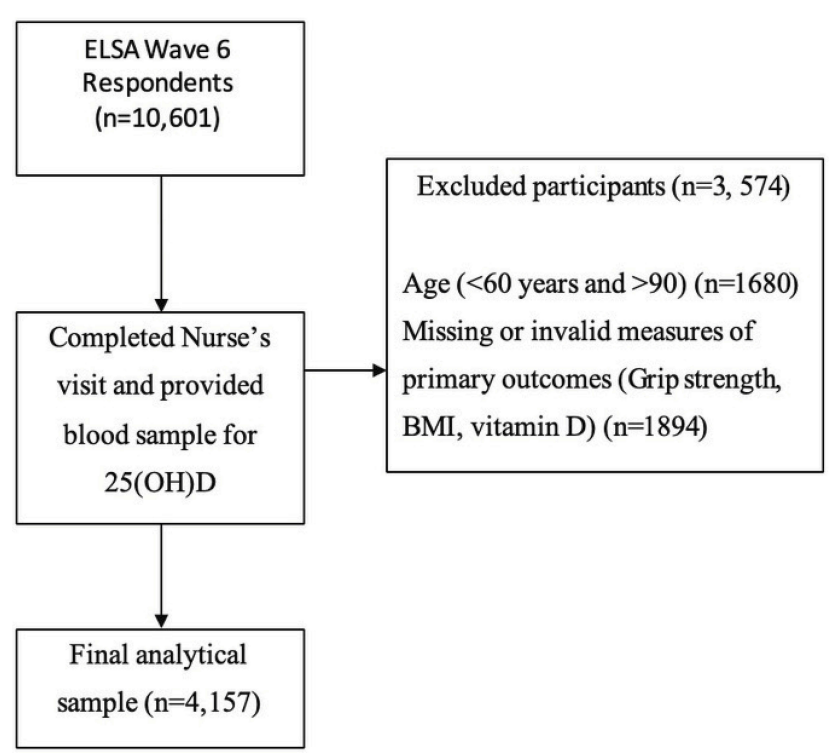

Figure I Participant enrolment in the study and reasons for exclusion.

\section{Measures Of Muscle Strength And Physical Function}

Handgrip strength was recorded as the mean grip strength of three readings using the participant's dominant hand. Gender and BMI-specific cut-offs were applied to categorize muscle weakness. ${ }^{1}$ Physical performance was measured using the short physical performance battery (SBBP). The $\mathrm{SPPB}^{25}$ is a composite battery of three physical tasks: an 8 foot $(2.44 \mathrm{~m})$ gait speed test, a repeated chair rise task and a static balance test and is supported by EWGSOP ${ }^{1}$ as a measure of physical performance for research. Individual tests scores were combined to give a composite SPPB score, where 0-6 represented poor physical performance, 7-9 "at-risk" and $>10$ represented high performers. ${ }^{25}$ We applied the cut-off of $0-6$ to indicate poor or impaired lower extremity performance. Falls rate was reported as one fall or multiple falls in past 12 months; medical attention sought in relation to the fall was also noted.

\section{Potential Confounders And Related Variables}

Background socio-demographic, health status, lifestyle vitamin D and muscle function related data were recorded. This included: gender, educational attainment [minimum of O-level (secondary school) education achieved] and employment status (employed/retired). Health-related factors; self-reported health (compared to others of the same age); excellent, very good, good, fair and poor. Self-reported long-standing illness (Yes/No), whether the self-reported longstanding illness was limiting (Yes/No) and medication use (polypharmacy defined as taking 5 or more medications daily). BMI and waist circumference were recorded. Lifestyle factors included physical activity categorized as mild, moderate and vigorous activity more than once per week. Alcohol intakes, high alcohol frequency (drinks alcohol most days), and current smoking status (Yes/No). Health behaviors which may influence serum 25(OH)D status in the ELSA dataset were season of blood sampling, sun holiday within the past 12 months (Yes/No) and vitamin D-containing supplement use, available as prescribed vitamin $\mathrm{D}$ and calcium for the treatment of osteoporosis. Vitamin D intake from non-prescribed vitamin D supplements or from diet was not available.

\section{Data Analysis And Statistics}

Descriptive statistics are presented as means and SD or counts and proportions for demographic and lifestyle characteristics. Normality was assessed visually using histogram matrix plots and ladder tables to identify appropriate transformations, ${ }^{29}$ and Monte Carlo simulations to identify outliers. ${ }^{30}$ Multiple logistic regression analysis was used to explore the determinants of poor physical performance and poor muscle strength and presented as odds ratios (OR) and 95\% confidence intervals (CI). We applied multivariate analysis of variance (MANOVA) to all explanatory variables to test the assumptions of the model, before including them in a stepwise regression. Only variables that reached statistical significance in the initial analysis were considered for the models and after testing for collinearity by scatter plots and Variance Inflation Factors Tests for linear relationships. Tests were performed by the inclusion of continuous predictor variables to the model and were reported as OR for a single unit change with 95\% CI. In adjusted models, covariates were selected based on previous research findings and following stepwise regression to test the contribution of each predictor (excluded Pr. $>0.25$ ). The contribution of covariates to muscle function was examined by including each set of covariates in the following order; model 1; poor physical performance defined as a score of $0-6$ in the SPPB, sociodemographic factors, health-related behaviors, physical factors and health conditions. In model 2, low HGS strength defined by gender and BMI-specific cut-offs. Corresponding unadjusted results are also reported. All analyses were performed in STATA 14.0 (Stata Corp LP).

\section{Results}

\section{Characteristics Of Study Population}

The present study comprised 4157 community-dwelling adults with a mean age of 69.8 (SD 6.9) years. Demographic, 
lifestyle and health characteristics are presented in Table 1. The prevalence of polypharmacy was $29.1 \%$ and longstanding limiting disease was $54.3 \%$, though most participants (76.3\%) rated their health as "good" or above. Overweight and obesity were common $(73 \%)$, while $41.8 \%$ of participants reported partaking regularly in vigorous physical activity.

Mean serum 25(OH)D concentration was 49.7 (SD 23.5) $\mathrm{nmol} / \mathrm{L}$ (Table 1). Over half of participants (53.7\%) had serum $25(\mathrm{OH}) \mathrm{D}$ concentrations $<50 \mathrm{nmol} / \mathrm{L}$ (insufficient) of which $21.8 \%$ were vitamin $\mathrm{D}$ deficient $(<30 \mathrm{nmol} / \mathrm{L})$. Vitamin D supplement use was low at $5.1 \%$, though only prescribed vitamin $\mathrm{D}$ for the treatment of osteoporosis was available. Almost half (48.6\%) of the study group reported sun holiday travel within the previous 12 months.

\section{Prevalence Of Impaired Muscle Strength, Poor Physical Performance And Falls}

In the present study, $30.6 \%$ of older adults had low HGS, as classified by gender and BMI-specific cut-offs (Table 2). Poor physical performance (SPPB $\leq 6)$ was identified in $12.7 \%$, with a further 19.3\% classed as "at-risk" (SPPB 7-9); most participants (67.9\%) were considered high performers with scores of 10-12. A single fall or multiple falls occurred in $15.8 \%$ and $10.5 \%$, respectively, in the past 12 months. The prevalence of impaired muscle strength, reduced performance and falls was consistently higher in women than men (Table 2).

\section{Muscle Strength And Physical Performance According To Serum 25 $(\mathrm{OH}) \mathrm{D}$ Concentration}

Low HGS and SPPB according to 25(OH)D quintiles are presented in Figures 2 and 3 and Table 3. The lowest quintile (Q1, $\leq 28 \mathrm{nmol} / \mathrm{L})$ was compatible with vitamin D deficiency, while the highest quintiles, Q4 and Q5, were compatible with sufficiency at 25(OH)D $>50 \mathrm{nmol} / \mathrm{L}$. In Q1, 40.4\% of older adults had impaired HGS compared with $25.2 \%$ and $26.8 \%$ in Q4 and Q5, respectively. Consistent with this, the prevalence of poor physical performance significantly decreased in line with increasing 25(OH)D concentration (Figures 2 and 3). Poor performance (SPPB) was almost three-fold higher $(21.6 \%)$ in $\mathrm{Q} 1(\leq 28 \mathrm{nmol} / \mathrm{L})$ compared with levels $\geq 50$ $\mathrm{nmol} / \mathrm{L}$ (7.9-9.0\%). In contrast, the rate of recurrently falls did not significantly differ between vitamin D deficiency (Q1) and sufficiency (Q5) at $11.6 \%$ and $9.9 \%$, respectively (Table 3 ).

\section{Factors Associated With Low SPPB In Community-Dwelling Older Adults}

After adjusting for pre-defined covariates, multivariate logistic regression analysis (Table 4) supported the findings that poor physical performance (SPPB score $\leq 6)$ was significantly associated with vitamin D deficiency $(<30 \mathrm{nmol} / \mathrm{L})$ [OR $1.65(1.31,2.09), \mathrm{p}<0.001]$. Regular moderate physical activity [OR $0.30(0.24,0.38), p<0.001]$ was the only variable significantly associated with lower odds of poor physical performance. BMI, however, was not a statistically significant correlate of poor physical performance, unadjusted OR for obese BMI was $1.87(1.46,2.39, p<0.001)$, but this was no longer statistically significant [OR $1.03(0.71,1.25)$, $p=0.84]$ on adjusted analysis. Other determinants of impaired SPPB included advanced age ( $80+$ years), female gender, being retired, poor self-reported health, falls $(>1)$ and polypharmacy.

\section{Factors Associated With Poor Muscle Strength In Community-Dwelling Older Adults}

After adjusting for pre-defined covariates, multivariate logistic regression analysis (Table 5) showed that vitamin D deficiency $(<30 \mathrm{nmol} / \mathrm{L})$ was a significant determinant of impaired HGS [OR $1.44 \quad(1.22,1.71)$ p $<0.001]$. Consistent with the SPPB findings, engaging in moderate physical activity was the only positive correlate of muscle strength identified [OR $0.65(0.58,0.79)]$. The model showed that aged $70+$, female gender, retired, fair selfreported health, falls $>1$, polypharmacy and overweight or obesity were significantly associated with low muscle strength.

\section{Discussion}

Vitamin D deficiency [25(OH)D $<30 \mathrm{nmol} / \mathrm{L}$ ] was significantly associated with both reduced muscle strength and physical performance in a large sample $(n=4157)$ of community-dwelling adults aged 60 years and over, as determined by handgrip strength and the Short Physical Performance Battery, respectively. Given that vitamin D deficiency is prevalent in older populations ${ }^{12,13}$ focusing on correcting deficiency at the $30 \mathrm{nmol} / \mathrm{L}$ cut-off ${ }^{11}$ may be important for protecting muscle function in ageing, in addition to the established effects on preventing bone disease. ${ }^{27}$ We identified impaired muscle strength and physical performance in $30.6 \%$ and $12.7 \%$, respectively, of community-dwelling adults aged 60 and over. This is 
Table I Characteristics Of The Study Population Overall And By Gender ( $n=4 \mid 57)$

\begin{tabular}{|c|c|c|c|c|c|c|}
\hline & \multicolumn{2}{|c|}{ Overall $(n=4 \mid 57)$} & \multicolumn{2}{|c|}{ Males $(n=1874)$} & \multicolumn{2}{|c|}{ Females $(n=2283)$} \\
\hline & $\mathbf{N}(\%)$ & SD & $\mathbf{N}(\%)$ & SD & $\mathbf{N}(\%)$ & SD \\
\hline \multicolumn{7}{|l|}{ Socio-demographic factors } \\
\hline Age, mean, SD, years & 69.8 & 6.9 & 69.7 & 7.0 & 69.8 & 6.9 \\
\hline $60-69$ & $2268(54.6)$ & & $1033(55.1)$ & & $1235(54.1)$ & \\
\hline $70-79$ & $1450(34.9)$ & & $64 \mid(34.2)$ & & $809(35.4)$ & \\
\hline $80+$ & $439(10.6)$ & & $200(10.7)$ & & $239(10.5)$ & \\
\hline${ }^{\mathrm{a} E d u c a t i o n} \geq \mathrm{O}$ level & $3099(74.9)$ & & I483 (79.5) & & $1616(71.1)$ & \\
\hline Employment - retired & $3090(74.3)$ & & $1362(72.7)$ & & $1728(75.7)^{* * *}$ & \\
\hline Ethnicity (non-white) & $87(2.1)$ & & $46(2.5)$ & & $41(1.8)$ & \\
\hline \multicolumn{7}{|l|}{ Health and lifestyle factors } \\
\hline BMI kg/m², mean, SD & 28.0 & 4.8 & 27.9 & 4.1 & 28.1 & 5.3 \\
\hline bUnder $(<18.5)$ & $37(0.9)$ & & $7(0.4)$ & & $30(1.3)^{* * *}$ & \\
\hline Normal $(\geq 18.5$ to $<25)$ & $1085(26.1)$ & & $432(23.1)$ & & $653(28.6)^{* * *}$ & \\
\hline Overweight $(\geq 25$ to $<30)$ & $1823(43.9)$ & & $940(50.2)$ & & $883(38.7)^{* * *}$ & \\
\hline Obese $(\geq 30)$ & $1212(29.1)$ & & $495(26.4)$ & & $717(31.4)^{* * *}$ & \\
\hline \multicolumn{7}{|l|}{ Physical activity } \\
\hline Vigorous (>I/wk) & $1739(4 \mid .8)$ & & $923(49.3)$ & & $816(35.7)^{* * *}$ & \\
\hline Moderate $(>1 / w k)$ & $2703(65.0)$ & & $1283(68.5)$ & & $1420(62.2)^{* * *}$ & \\
\hline Mild (>I/wk) & $3463(83.3)$ & & $1450(77.4)$ & & $2013(88.2)^{* * *}$ & \\
\hline Current smoker & $391(9.4)$ & & $174(9.3)$ & & $217(9.5)$ & \\
\hline Alcohol - 5-6 days/week & $254(6.1)$ & & I 48 (7.9) & & $106(4.6)^{* * *}$ & \\
\hline \multicolumn{7}{|l|}{ SR health } \\
\hline Excellent & $485(11.7)$ & & $212(11.3)$ & & $273(11.9)$ & \\
\hline Very good & $1323(3 \mid .8)$ & & $607(32.4)$ & & $716(31.4)$ & \\
\hline Good & $1365(32.8)$ & & $617(32.9)$ & & $748(32.8)$ & \\
\hline Fair & $770(18.5)$ & & $346(18.5)$ & & $424(18.6)$ & \\
\hline Poor & $213(5.1)$ & & $92(4.9)$ & & $|2|(5.3)$ & \\
\hline Long standing illness & $2258(54.3)$ & & $1007(53.7)$ & & $|25|(54.8)$ & \\
\hline${ }^{c}$ Limiting & $1328(58.8)$ & & $557(55.3)$ & & $771(61.7)^{* *}$ & \\
\hline Polypharmacy ( $\geq 5$ meds) & $1209(29.1)$ & & $548(29.2)$ & & $661(28.9)$ & \\
\hline Poor QoL (CASP <36) & $772(18.6)$ & & $378(20.2)$ & & $394(17.3)^{*}$ & \\
\hline \multicolumn{7}{|l|}{ Vitamin D related factors } \\
\hline 25(OH)D nmol/L, mean, SD & 49.7 & 23.5 & 50.5 & 23.1 & $49.1 *$ & 23.8 \\
\hline \multicolumn{7}{|l|}{ Low 25(OH)D status } \\
\hline$<30 \mathrm{nmol} / \mathrm{L}$ & $908(21.8)$ & & $359(19.2)$ & & $549(24.1)^{* * *}$ & \\
\hline$<50 \mathrm{nmol} / \mathrm{L}$ & $2234(53.7)$ & & $977(52.1)$ & & $1257(55.1)$ & \\
\hline${ }^{d}$ VitD supp use, $n(\%)$ & $212(5.1)$ & & $24(1.3)$ & & $188(8.2)^{* * *}$ & \\
\hline${ }^{\mathrm{e}}$ Sun holiday travel, $\mathrm{n}(\%)$ & $1904(48.6)$ & & $858(48.9)$ & & $1046(48.4)$ & \\
\hline
\end{tabular}

Notes: Results are presented as means and standard deviation (SD) or counts and proportions, $\mathrm{n}$ (\%). ${ }^{\mathrm{a}} \mathrm{O}$-level, approximately equivalent to secondary school. ${ }^{\mathrm{b}} \mathrm{BMI}$ categories. ${ }^{\mathrm{C}}$ If the long-standing illness is limiting. ${ }^{\mathrm{d} V i t a m i n} \mathrm{D}$ supplement use, with or without calcium, prescribed for osteoporosis. ${ }^{\mathrm{e}} \mathrm{Travel}$ abroad within the previous $\mathrm{I} 2$ months, yes or no. $* * * * * *$ Denote statistically significant differences between groups: $* \mathrm{P}<0.05, * * \mathrm{P}<0.0 \mathrm{I}, * * * \mathrm{P}<0.00 \mathrm{I}$.

Abbreviations: $\mathrm{kg} / \mathrm{m}^{2}$, kilograms per meter squared; SR, self-reported; CASP-19, Control, Autonomy, Self-realization and Pleasure, a four domain older-adult quality of life measure. 
Table 2 HGS, SPPB And Falls In Community-Dwelling Older Adults $(n=4157)$

\begin{tabular}{|c|c|c|c|}
\hline & $\begin{array}{l}\text { Overall } \\
(n=4 \mid 57)\end{array}$ & $\begin{array}{l}\text { Males } \\
(n=1874)\end{array}$ & $\begin{array}{l}\text { Females } \\
(n=2283)\end{array}$ \\
\hline & $\mathbf{N}(\%)$ & $\mathbf{N}(\%)$ & $\mathbf{N}(\%)$ \\
\hline${ }^{\mathrm{a}}$ Low HGS \% & $|27|(30.6)$ & $505(26.9)$ & $766(33.6)^{* * *}$ \\
\hline SPPB poor $(\leq 6)$ & $527(12.7)$ & $192(10.3)$ & $335(14.7)^{* * *}$ \\
\hline At-risk (7-9) & $804(19.3)$ & $340(18.1)$ & $464(20.3)^{* * *}$ \\
\hline Best $(10-12)$ & $2826(67.9)$ & I342 (7I.6) & $1484(65.0)$ \\
\hline${ }^{\mathrm{b}}$ Falls one fall & $658(15.8)$ & $267(14.3)$ & $391(17.1)^{* *}$ \\
\hline Multiple falls $(>1)$ & $438(10.5)$ & $173(9.2)$ & $265(11.6)^{* *}$ \\
\hline${ }^{c}$ Medical attention & $813(74.2)$ & $354(80.5)$ & $459(69.9)^{* * *}$ \\
\hline
\end{tabular}

Notes: ${ }^{a}$ Classified by gender and BMI-specific cut-offs. ${ }^{b}$ Fall in the past 12 months. Medical attention was sought for fall. $* *, * * *$ Denote statistically significant differences between groups: $* * P<0.01, * * * P<0.001$.

Abbreviations: HGS, handgrip strength; SPPB, short physical performance battery.

broadly in line with the prevalence of sarcopenia, estimated at 1-29\%, in community-dwelling populations aged 50 years and over. ${ }^{31}$ The prevalence and incidence of sarcopenia is reported to increase further in hospitalized and long-term care for older adult populations. ${ }^{31,32}$ It should be noted, however, that the present study focused on impaired muscle function only and not specifically on sarcopenia (since muscle mass was not available in the dataset). Muscle function, however, is considered a principal determinant of sarcopenia in recently updated definitions. ${ }^{2}$

Based on the analysis by vitamin $\mathrm{D}$ quintiles, older adults with the lowest serum $25(\mathrm{OH}) \mathrm{D}$, had the highest prevalence of impaired muscle strength and performance (40.4\% and $25.2 \%$ ) compared with people with adequate levels $(21.6 \%$ and $7.9 \%)$. This finding was further supported by the regression analysis, which showed that vitamin D deficiency ( $<30 \mathrm{nmol} / \mathrm{L}$ ) was significantly associated with both poor physical performance and low handgrip strength. Of note, engagement in moderate physical activity was associated with lower odds of impaired muscle strength and low SPPB, which is in line with the evidence supporting physical activity as a modifiable factor in preserving muscle mass, strength and potentiating muscle protein synthesis., 6 Other factors associated with low HGS and low SPPB included advanced age, female gender and variables indicative of poorer health status such as lower self-rated health, polypharmacy and recurrent falls reflecting the complex and multifaceted nature of age-related muscle impairment. Our findings identify vitamin D deficiency as a potentially modifiable lifestyle factors associated with poor muscle function and further supports the established inverse association with physical activity. 6,10

Falls were not significantly associated with $25(\mathrm{OH}) \mathrm{D}$ concentrations in the present study, though this was a

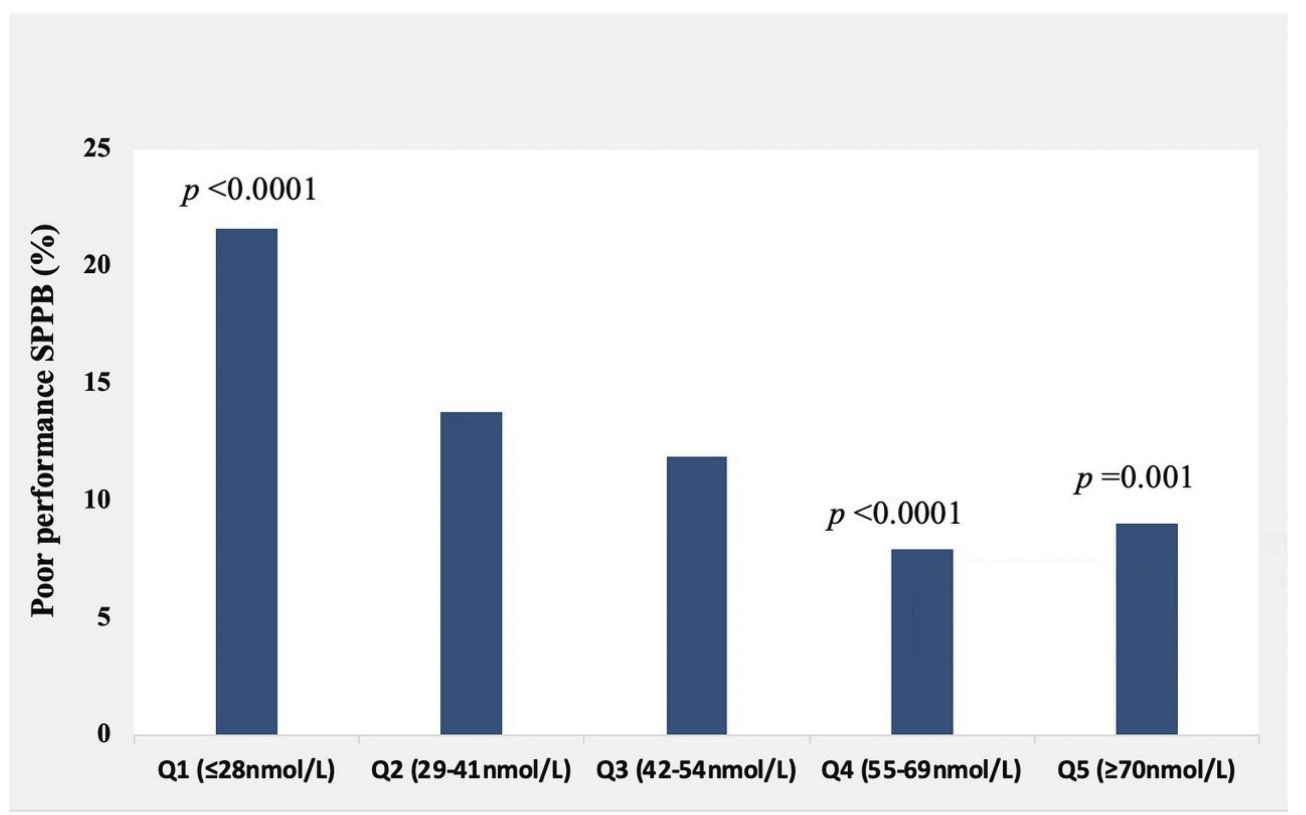

Figure 2 Prevalence of poor physical performance (SPPB $\leq 6)$ across serum $25(\mathrm{OH}) \mathrm{D}$ quintiles, $\mathrm{n}=527$. There was a significantly higher proportion of older adults with low SPPB in QI [25(OH)D $\leq 28 \mathrm{nmol} / \mathrm{L}$, vitamin $D$ deficient] relative to $\mathrm{Q} 4$ and 5 [vitamin sufficiency $25(\mathrm{OH}) \mathrm{D}>50 \mathrm{nmol} / \mathrm{L}$ ]. 


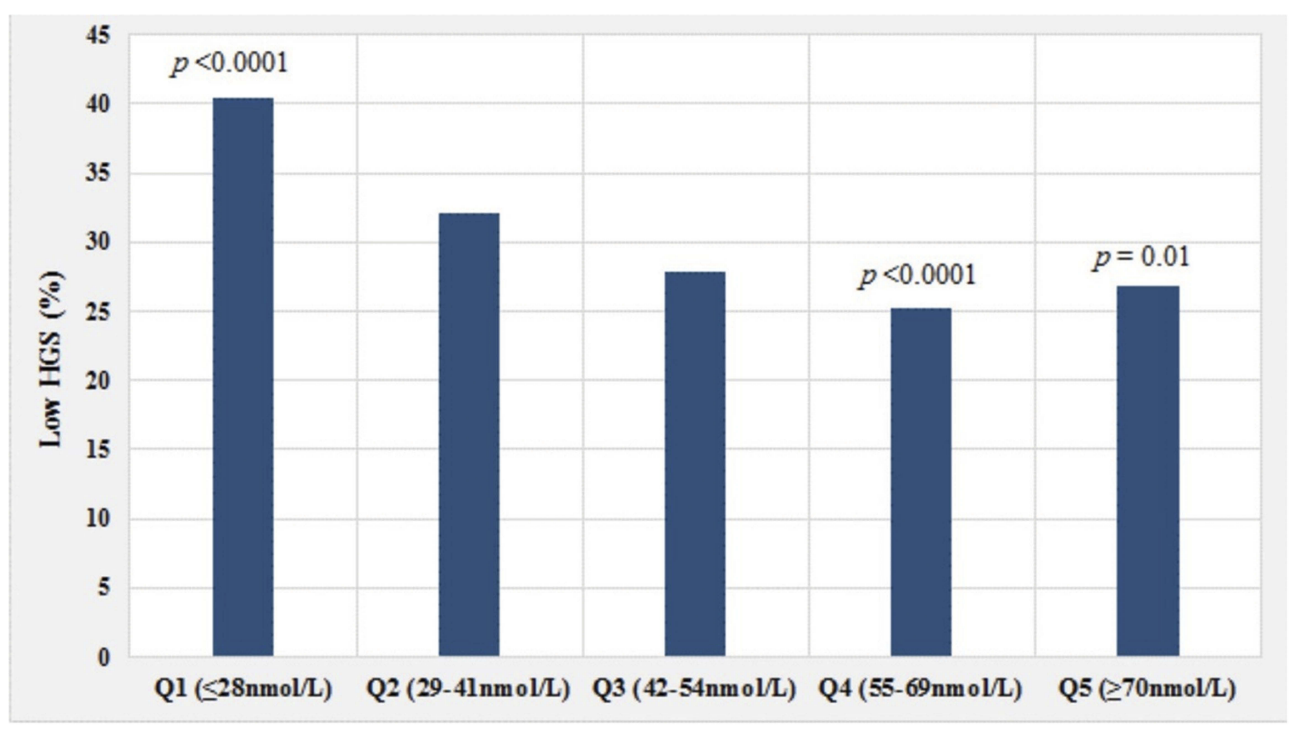

Figure 3 Prevalence of poor muscle strength (low HGS) across serum $25(\mathrm{OH}) \mathrm{D}$ quintiles, $\mathrm{n}=127 \mathrm{I}$. There was a significantly higher prevalence of impaired muscle strength in QI [25(OH)D $\leq 28 \mathrm{nmol} / \mathrm{L}$, vitamin $D$ deficient] relative to quintiles 4 and 5 [vitamin $D$ sufficiency $25(\mathrm{OH}) \mathrm{D}>50 \mathrm{nmol} / \mathrm{L}]$.

secondary aim. The occurrence of single or multiple falls was $15.8 \%$ and $10.5 \%$, respectively, in the past year but did not significantly differ by $25(\mathrm{OH}) \mathrm{D}$ status. A Cochrane review, ${ }^{22}$ and similarly a recent large $\mathrm{RCT},{ }^{33}$ showed no effect for vitamin D supplementation on falls in the community, however, supplementation in older adult subgroups with vitamin $\mathrm{D}$ deficiency might protect against falls. ${ }^{22}$ Findings from a recent Cochrane review suggested that vitamin $\mathrm{D}$ supplementation may reduce falls rate in residential care facilities, ${ }^{21}$ which represent a more dependent and vulnerable group compared with community-living populations in the present study. While vitamin $\mathrm{D}^{21}$ and sarcopenia ${ }^{3}$ have been implicated in falls risk, it is acknowledged that falls in ageing have a complex and multifactorial etiology involving health, sensory, medication and living environmental risk factors. ${ }^{21,22}$

To date, intervention studies into the effects of vitamin D supplementation on skeletal muscle in ageing have yielded mixed findings ${ }^{8,10,18-20}$ though a trend towards improved lower extremity muscle performance is emerging. Lower limb function is commonly assessed by practical and clinical measures such as gait speed or the Timed-Up-and-Go test (TUG). The SPPB, applied in the present study, is a measure of physical performance of the lower extremities, acting as a comprehensive indicator of balance, gait, muscle strength and endurance. ${ }^{1}$ We applied a restrictive score of $<6$ for the SPPB for muscle impairment and vitamin D deficiency of $<30 \mathrm{nmol} / \mathrm{L}$. A recent intervention study among older adults

Table 3 Low Handgrip Strength And Poor Muscle Performance According To Serum 25(OH)D Quintiles In Community-Dwelling Older Adults

\begin{tabular}{|c|c|c|c|c|c|}
\hline 25(OH)D Quintiles nmol/L & Q I $\leq \mathbf{2 8}$ & Q2 29-4I & Q3 42-54 & Q4 55-69 & Q5 $\geq 70$ \\
\hline $\mathrm{n}, \%$ & $856(20.6)$ & $836(20.1)$ & $845(20.3)$ & $821(19.8)$ & $799(19.2)$ \\
\hline Low HGS \% & $346(40.4)^{* * *}$ & $268(32.1)$ & $236(27.9)$ & $207(25.2)^{* * *}$ & $214(26.8)^{* *}$ \\
\hline \multicolumn{6}{|l|}{ SPPB (0-12) } \\
\hline Poor $(\leq 6)$ & $185(21.6)^{* * *}$ & $115(13.8)$ & $90(11.9)$ & $65(7.9)^{* * *}$ & $72(9.0)^{* * *}$ \\
\hline At risk (7-9) & $200(23.4)^{* * *}$ & $|7|(20.5)$ & $149(17.6)$ & I46 (I7.8) & $138(17.3)$ \\
\hline High $(\geq 10)$ & $47 \mid(55)^{* * *}$ & $550(65.8)$ & $606(71.7)$ & $610(74.3)^{* * *}$ & $589(73.7)^{* * *}$ \\
\hline${ }^{\mathrm{a}}$ Falls $(>1)$ & $99(11.6)$ & $86(10.3)$ & $84(9.9)$ & $90(10.9)$ & $79(9.9)$ \\
\hline
\end{tabular}

Notes: a Falls, reported to have had multiple $(>I)$ falls in past 12 months. $* * * * *$ Denote statistically significant differences between groups: $* * P<0.01, * * * P<0.00 \mathrm{I}$. Abbreviations: Q, quintiles; low HGS, low handgrip strength established by BMI and gender-specific cut-offs; SPPB, short physical performance battery. 
Table 4 Logistic Regression Analysis Showing Unadjusted And Adjusted Odds Ratios (95\% Cl) For Poor Physical Performance (SPPB $\leq 6)$ In Community-Dwelling Older Adults ( $n=4157)$

\begin{tabular}{|c|c|c|c|c|c|c|}
\hline & \multicolumn{3}{|c|}{ Unadjusted Poor SPPB $(\leq 6)$} & \multicolumn{3}{|c|}{ Adjusted Poor SPPB $(\leq 6)$} \\
\hline & OR & $(95 \% \mathrm{Cl})$ & $P$ & OR & $(95 \% \mathrm{Cl})$ & $P$ \\
\hline Female & 1.50 & $(1.25,1.82)$ & $<0.001$ & 1.39 & $(1.11,1.74)$ & 0.004 \\
\hline Age 60-69 (reference) & - & - & 1 & - & - & I \\
\hline $70-79$ & 1.86 & $(1.49,2.31)$ & $<0.001$ & 1.29 & $(0.99,1.69)$ & 0.050 \\
\hline $80-89$ & 7.38 & $(5.76,9.47)$ & $<0.001$ & 3.78 & $(2.78,5.15)$ & $<0.001$ \\
\hline Employment-retired & 4.78 & $(2.94,7.69)$ & $<0.001$ & 1.98 & $(1.18,3.34)$ & 0.010 \\
\hline Vitamin D deficiency (<30nmol/L) & 2.43 & $(1.99,2.95)$ & $<0.001$ & 1.65 & $(1.31,2.09)$ & $<0.001$ \\
\hline${ }^{\mathrm{a}}$ Vitamin $\mathrm{D}$ supplement use & 2.10 & $(1.56,2.88)$ & $<0.001$ & 1.37 & $(0.91,2.05)$ & 0.134 \\
\hline Self-reported health - poor & 3.25 & $(2.67,3.96)$ & $<0.001$ & 1.55 & $(1.22,1.97)$ & $<0.001$ \\
\hline Physical activity-moderate & 0.16 & $(0.13,0.19)$ & $<0.001$ & 0.30 & $(0.24,0.38)$ & $<0.001$ \\
\hline${ }^{\mathrm{b}}$ Falls $(>1)$ & 3.78 & $(3.00,4.75)$ & $<0.001$ & 2.55 & $(1.94,3.36)$ & $<0.001$ \\
\hline Polypharmacy ( $\geq 5$ medications/d) & 4.90 & $(4.1,5.9)$ & $<0.001$ & 2.42 & $(1.93,3.02)$ & $<0.001$ \\
\hline Low HGS & 5.10 & $(4.2,6.1)$ & $<0.001$ & 2.17 & $(1.73,2.72)$ & $<0.001$ \\
\hline Poor QoL (CASP-19 <36) & 1.95 & $(1.58,2.39)$ & $<0.001$ & 1.25 & $(0.97,1.61)$ & 0.080 \\
\hline BMI - obese & 1.87 & $(1.46,2.39)$ & $<0.001$ & 1.03 & $(0.7 \mathrm{I}, \mathrm{I} .25)$ & 0.84 \\
\hline
\end{tabular}

Notes: a Use as prescribed treatment of osteoporosis. ${ }^{b}$ Falls, reported as multiple ( $>$ I) falls in past 12 months. Reference category: male, age $60-69$; employed, $25(\mathrm{OH}) \mathrm{D}$ $>30 \mathrm{nmol} / \mathrm{L}$, no vitamin D supplement use, excellent self-reported health; low physical activity, no falls, no polypharmacy, i.e. medications <4/d, HGS in normal range by gender and BMI classification, CASP >36, BMI in the underweight category. Adjusted analysis adjusted for age, gender, and physical activity. All models included the same variables and categorizations/groupings, for visual presentation only significant variable categories are presented.

Abbreviations: OR, odds ratio; Cl, confidence interval; HGS, handgrip strength; QoL, quality of life; CASP-19, Control, Autonomy, Self-realisation, Pleasure, which is a 4 domain QoL measure for older adults.

with impaired muscle function, defined as SPPB $\leq 9$ and vitamin $\mathrm{D}$ deficiency of $25(\mathrm{OH}) \mathrm{D}$ less than $50 \mathrm{nmol} / \mathrm{L}$, showed that a vitamin $\mathrm{D}$ and protein supplement reduced intramuscular fat and improved muscle density compared with placebo. ${ }^{10}$ Future intervention studies into the effects of reversing vitamin $\mathrm{D}$ deficiency at $30 \mathrm{nmol} / \mathrm{L}$ level on

Table 5 Logistic Regression Analysis Showing Unadjusted And Adjusted Odds Ratios (95\% Cl) For Low Handgrip Strength In Community-Dwelling Older Adults ( $n=4157)$

\begin{tabular}{|c|c|c|c|c|c|c|}
\hline & \multicolumn{3}{|c|}{ Unadjusted Low HGS } & \multicolumn{3}{|c|}{ Adjusted Low HGS } \\
\hline & OR & $(95 \% \mathrm{Cl})$ & $\mathbf{P}$ & OR & $(95 \% \mathrm{Cl})$ & $P$ \\
\hline Female & 1.37 & $(1.19,1.57)$ & $<0.001$ & 1.25 & $(1.08,1.46)$ & 0.003 \\
\hline 70-79 & 2.32 & $(2.00,2.69)$ & $<0.001$ & 1.92 & $(1.62,2.26)$ & $<0.001$ \\
\hline $80-89$ & 7.49 & $(6.00,9.35)$ & $<0.001$ & 4.71 & $(3.69,6.02)$ & $<0.001$ \\
\hline Employment-retired & 2.81 & $(2.19,3.62)$ & $<0.001$ & 1.49 & $(1.15,1.96)$ & 0.003 \\
\hline Vitamin D deficiency $(<30)$ & 1.79 & $(1.54,2.09)$ & $<0.001$ & 1.44 & $(1.21,1.71)$ & $<0.001$ \\
\hline Self-reported health - poor & 2.20 & $(1.87,2.59)$ & $<0.001$ & 1.39 & $(1.15,1.67)$ & 0.001 \\
\hline Physical Activity - Moderate & $0.4 I$ & $(0.36,0.48)$ & $<0.001$ & 0.68 & $(0.58,0.79)$ & $<0.001$ \\
\hline${ }^{\mathrm{a}}$ Falls $(>1)$ & 2.08 & $(1.69,2.54)$ & $<0.001$ & 1.47 & $(1.17,1.86)$ & 0.001 \\
\hline Polypharmacy ( $\geq 5$ medications/d) & 2.63 & $(2.29,3.03)$ & $<0.001$ & $\mid .51$ & $(1.28,1.78)$ & $<0.001$ \\
\hline Poor SPPB ( $\leq 6$ points) & 5.07 & $(4.18,6.15)$ & $<0.001$ & 2.22 & $(1.77,2.77)$ & $<0.001$ \\
\hline Poor QoL (CASP-19, <36) & 1.36 & $(1.16,1.61)$ & $<0.001$ & 0.97 & $(0.8 I, I .17)$ & 0.785 \\
\hline BMI overweight & 1.29 & $(1.09,1.55)$ & 0.003 & 1.31 & $(1.08,1.59)$ & 0.006 \\
\hline Obese & 2.42 & $(2.02,2.91)$ & $<0.001$ & 2.26 & $(1.84,2.78)$ & $<0.001$ \\
\hline
\end{tabular}

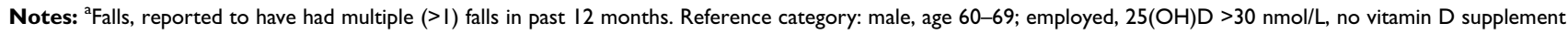
use, excellent self-reported health; low physical activity, no falls, no polypharmacy, i.e. medications $<4 / \mathrm{d}$, SPPB $>6$, CASP $>36$, BMI in the underweight category. Adjusted analysis adjusted for age, gender, and physical activity. All models included the same variables and categorizations/groupings, for visual presentation only significant variable categories are presented.

Abbreviations: OR, odds ratio; Cl, confidence interval; SPPB, short physical performance battery; QoL, quality of life; CASP-I9, Control, Autonomy, Self-realization, Pleasure, a four domain QoL measure for older adults. 
comprehensive muscle outcomes among vulnerable older adults in the community would be of interest.

In the present study, we identified that older people in the lowest quintile of vitamin D (compatible with deficiency), had the poorest muscle strength and physical performance and represent an important group to target for interventions to reverse deficiency. The recent EWGSOP updated consensus paper on sarcopenia emphasizes the public health and clinical importance of maintaining muscle function in ageing. ${ }^{2}$ The threshold of $<30 \mathrm{nmol} / \mathrm{L}$ for vitamin $\mathrm{D}$ deficiency is established for adverse effects on bone health ${ }^{27}$ and may also accelerate age-related muscle decline. The recent Vitamin D Assessment (ViDA) study showed beneficial effects of vitamin D supplementation in aged $50+$ adults for intermediate outcomes such as bone mineral density, but not for falls, and only in people with $25(\mathrm{OH}) \mathrm{D}<30 \mathrm{nmol} / \mathrm{L}{ }^{33}$ The low usage of vitamin D supplements at 5.1\%, which was prescribed for bone health in the present study, is compatible with other community-dwelling cohorts ${ }^{12}$ and was not significantly associated with either impaired physical performance or muscle strength. This suggests that eliminating vitamin D deficiency in older populations may require other strategies such as food fortification approaches. ${ }^{34,35}$ Focusing interventions on older people most at risk of vitamin D deficiency $(<30 \mathrm{nmol} / \mathrm{L})^{12,13}$ seems sensible, even though such sub-groups may be "hard to reach" in society and to engage in clinical trials. Theses subgroups may include, for example, the oldest old, those with poorer health status, of non-white ethnicity, living alone or living with socioeconomic disadvantage. The latter may be particularly important, given that the evidence is currently deemed inadequate to make practical recommendations for vitamin D supplementation for the prevention of age-related loss of muscle mass and function. ${ }^{6}$

The study had several strengths, for example, the findings are derived from a large $(n=4157)$ well-defined population of community-dwelling older people aged 60 years and over with comprehensive assessments and measures. Moreover, handgrip strength and a composite measure of muscle performance (SPPB) were used, both supported by the EWGSOP. ${ }^{1}$ A number of limitations need to be mentioned, namely serum $25(\mathrm{OH}) \mathrm{D}$ in the ELSA cohort was assessed by immunoassay (DiaSorin), while the gold standard is considered LC-MS/MS. Analysis, however, was conducted in a laboratory which is a member of DEQAS, reported CVs and QCs were in the expected range and consistent with data from ELSA previously published..$^{10,36,37}$ Furthermore, in line with the study design, our findings cannot demonstrate cause and effect, and future intervention studies conducted in vitamin D deficient older populations would be required.

In conclusion, impaired muscle strength and physical performance were identified in $30.6 \%$ and $12.7 \%$, respectively, of older adults, rising to $40.4 \%$ and $25.2 \%$ amongst those with the lowest vitamin D concentrations. Consistent with this, vitamin D deficiency $[25(\mathrm{OH}) \mathrm{D}<30 \mathrm{nmol} / \mathrm{L}]$ was associated with both impaired muscle strength and impaired physical performance based on regression analysis models. It is accepted, that at a minimum, we should seek to eliminate vitamin $\mathrm{D}$ deficiency of $\leq 30 \mathrm{nmol} / \mathrm{L}$, the cut-off for the prevention of bone disease, this strategy that may also protect skeletal muscle function in ageing populations.

\section{Abbreviations}

ELSA, English Longitudinal Study of Aging; HGS, handgrip strength; SPPB, short physical performance battery.

\section{Author Contributions}

All authors contributed to data analysis, drafting and revising the article, gave final approval of the version to be published, and agree to be accountable for all aspects of the work.

\section{Funding}

This work was supported by an Irish Research Council postgraduate scholarship for NA.

\section{Disclosure}

The authors report no conflicts of interest in this work.

\section{References}

1. Cruz-Jentoft AJ, Baeyens JP, Bauer JM, et al. Sarcopenia: European consensus on definition and diagnosis: report of the European Working Group on Sarcopenia in Older People. Age Ageing. 2010;39(4):412423. doi:10.1093/ageing/afq034

2. Cruz-Jentoft AJ, Bahat G, Bauer J, et al. Sarcopenia: revised European consensus on definition and diagnosis. Age Ageing. 2019;48(1):16-31. doi:10.1093/ageing/afy169

3. Landi F, Liperoti R, Russo A, et al. Sarcopenia as a risk factor for falls in elderly individuals: results from the ilSIRENTE study. Clin Nutr. 2012;31(5):652-658. doi:10.1016/j.clnu.2012.02.007

4. Rizzoli R, Reginster JY, Arnal JF, et al. Quality of life in sarcopenia and frailty. Calcif Tissue Int. 2013;93(2):101-120. doi:10.1007/ s00223-013-9758-y

5. Benjumea AM, Curcio CL, Duque G, Gomez F. Dynapenia and sarcopenia as a risk factor for disability in a falls and fractures clinic in older persons. Open Access Maced J Med Sci. 2018;6(2):344-349. doi:10.3889/oamjms.2018.087

6. Dent E, Morley JE, Cruz-Jentoft AJ, et al. International clinical practice guidelines for sarcopenia (ICFSR): screening, diagnosis and management. J Nutr Health Aging. 2018;22(10):1148-1161. doi:10.1007/ s12603-018-1139-9 
7. Paddon-Jones D, Leidy H. Dietary protein and muscle in older persons. Curr Opin Clin Nutr Metab Care. 2014;17(1):5-11. doi:10.1097/MCO.0000000000000011

8. Antoniak AE, Greig CA. The effect of combined resistance exercise training and vitamin D3 supplementation on musculoskeletal health and function in older adults: a systematic review and metaanalysis. BMJ Open. 2017;7(7):e014619. doi:10.1136/bmjopen2016-014619

9. Bosaeus I, Rothenberg E. Nutrition and physical activity for the prevention and treatment of age-related sarcopenia. Proc Nutr Soc. 2016;75(2):174-180. doi:10.1017/S002966511500422X

10. Englund DA, Kirn DR, Koochek A, et al. Nutritional supplementation with physical activity improves muscle composition in mobilitylimited older adults, the VIVE2 study: a randomized, double-blind, placebo-controlled trial. J Gerontol A Biol Sci Med Sci. 2017;73 (1):95-101. doi:10.1093/gerona/glx141

11. Roth DE, Abrams SA, Aloia J, et al. Global prevalence and disease burden of vitamin D deficiency: a roadmap for action in low- and middle-income countries. Ann N Y Acad Sci. 2018;1430(1):44-79. doi:10.1111/nyas. 13968

12. Laird E, O'Halloran AM, Carey D, et al. The prevalence of vitamin D deficiency and the determinants of $25(\mathrm{OH}) \mathrm{D}$ concentration in older Irish adults: data from The Irish Longitudinal Study on Ageing (TILDA). J Gerontol A Biol Sci Med Sci. 2018;73(4):519-525. doi:10.1093/gerona/glx168

13. Aspell N, Laird E, Healy M, Shannon T, Lawlor B, O'Sullivan M. The prevalence and determinants of vitamin $\mathrm{D}$ status in community-dwelling older adults: results from the English Longitudinal Study of Ageing (ELSA). Nutrients. 2019;11(6):1253. doi:10.3390/ nu11061253

14. Ceglia L. Vitamin D and skeletal muscle tissue and function. Mol Aspects Med. 2008;29(6):407-414. doi:10.1016/j.mam.2008.07.002

15. Dewansingh P, Melse-Boonstra A, Krijnen WP, van der Schans CP, Jager-Wittenaar H, van Den Heuvel E. Supplemental protein from dairy products increases body weight and vitamin D improves physical performance in older adults: a systematic review and meta-analysis. Nutr Res. 2018;49:1-22. doi:10.1016/j.nutres.2017. 08.004

16. Shardell M, Semba RD, Kalyani RR, Hicks GE, Bandinelli S, Ferrucci L. Serum 25-hydroxyvitamin D, plasma klotho, and lowerextremity physical performance among older adults: findings from the InCHIANTI study. J Gerontol A Biol Sci Med Sci. 2015;70 (9):1156-1162. doi:10.1093/gerona/glv017

17. Visser M, Deeg DJ, Lips P. Longitudinal aging study A. Low vitamin $\mathrm{D}$ and high parathyroid hormone levels as determinants of loss of muscle strength and muscle mass (sarcopenia): the longitudinal aging study amsterdam. J Clin Endocrinol Metab. 2003;88(12):5766-5772. doi:10.1210/jc.2003-030604

18. Bolland MJ, Grey A, Avenell A. Effects of vitamin D supplementation on musculoskeletal health: a systematic review, meta-analysis, and trial sequential analysis. Lancet Diabetes Endocrinol. 2018;6 (11):847-858. doi:10.1016/S2213-8587(18)30265-1

19. Ranathunga R, Hill TR, Mathers JC, et al. No effect of monthly supplementation with $12000 \mathrm{IU}, 24000 \mathrm{IU}$ or $48000 \mathrm{IU}$ vitamin D3 for one year on muscle function: the vitamin D in older people study. J Steroid Biochem Mol Biol. 2018.

20. Stockton KA, Mengersen K, Paratz JD, Kandiah D, Bennell KL. Effect of vitamin D supplementation on muscle strength: a systematic review and meta-analysis. Osteoporos Int. 2011;22(3):859-871. doi:10.1007/s00198-010-1407-y
21. Cameron ID, Dyer SM, Panagoda CE, et al. Interventions for preventing falls in older people in care facilities and hospitals. Cochrane Database Syst Rev. 2018;9:CD005465.

22. Gillespie LD, Robertson MC, Gillespie WJ, et al. Interventions for preventing falls in older people living in the community. Cochrane Database Syst Rev. 2012;9:CD007146.

23. Sanders KM, Stuart AL, Williamson EJ, et al. Annual high-dose oral vitamin D and falls and fractures in older women: a randomized controlled trial. JAMA. 2010;303(18):1815-1822. doi:10.1001/ jama.2010.594

24. Sohl E, Heymans MW, de Jongh RT, et al. Prediction of vitamin D deficiency by simple patient characteristics. Am J Clin Nutr. 2014;99 (5):1089-1095. doi:10.3945/ajcn.113.076430

25. Guralnik JM, Simonsick EM, Ferrucci L, et al. A short physical performance battery assessing lower extremity function: association with self-reported disability and prediction of mortality and nursing home admission. J Gerontol. 1994;49(2):M85-M94. doi:10.1093/geronj/49.2.m85

26. Steptoe A, Breeze E, Banks J, Nazroo J. Cohort profile: the English longitudinal study of ageing. Int J Epidemiol. 2013;42(6):1640-1648. doi: $10.1093 / \mathrm{ije} / \mathrm{dys} 168$

27. Institute of Medicine. Dietary Reference Intakes for Calcium and Vitamin D. Washington DC: The National Academies Press; 2011.

28. Holick MF. Sunlight, UV-radiation, vitamin D and skin cancer: how much sunlight do we need? Adv Exp Med Biol. 2008;624:1-15. doi:10.1007/978-0-387-77574-6_1

29. Tukey JW. Exploratory Data Analysis. Reading, MA: AddisonWesley; 1977.

30. Hoaglin DC, Iglewicz B, Tukey JW. Performance of some resistent rules for outlier labeling. J Alzheimers Dis. 1986;81:991-999.

31. Cruz-Jentoft AJ, Landi F, Schneider SM, et al. Prevalence of and interventions for sarcopenia in ageing adults: a systematic review. Report of the International Sarcopenia Initiative (EWGSOP and IWGS). Age Ageing. 2014;43(6):748-759. doi:10.1093/ageing/ afu115

32. Martone AM, Bianchi L, Abete $\mathrm{P}$, et al. The incidence of sarcopenia among hospitalized older patients: results from the Glisten study. $J$ Cachexia Sarcopenia Muscle. 2017;8(6):907-914. doi:10.1002/ jcsm. 12224

33. Scragg RKR. Overview of results from the Vitamin D Assessment (ViDA) study. J Endocrinol Invest. 2019. doi:10.1007/s40618-01901056-Z

34. Hayes A, Cashman KD. Food-based solutions for vitamin D deficiency: putting policy into practice and the key role for research. Proc Nutr Soc. 2017;76(1):54-63. doi:10.1017/S0029 665116000756

35. Jaaskelainen T, Itkonen ST, Lundqvist A, et al. The positive impact of general vitamin $\mathrm{D}$ food fortification policy on vitamin $\mathrm{D}$ status in a representative adult Finnish population: evidence from an 11-y follow-up based on standardized 25-hydroxyvitamin D data. Am J Clin Nutr. 2017;105(6):1512-1520. doi:10.3945/ajen.116.151415

36. de Oliveira C, Hirani V, Biddulph JP. Associations between vitamin $\mathrm{D}$ levels and depressive symptoms in later life: evidence from the English Longitudinal Study of Ageing (ELSA). J Gerontol A Biol Sci Med Sci. 2018;73(10):1377-1382. doi:10.1093/gerona/glx130

37. de Oliveira C, Biddulph JP, Hirani V, Schneider IJC. Vitamin D and inflammatory markers: cross-sectional analyses using data from the English Longitudinal Study of Ageing (ELSA). J Nutr Sci. 2017;6:e1. doi:10.1017/jns.2016.37 


\section{Publish your work in this journal}

Clinical Interventions in Aging is an international, peer-reviewed journal focusing on evidence-based reports on the value or lack thereof of treatments intended to prevent or delay the onset of maladaptive correlates of aging in human beings. This journal is indexed on PubMed Central, MedLine, CAS, Scopus and the Elsevier

Bibliographic databases. The manuscript management system is completely online and includes a very quick and fair peer-review system, which is all easy to use. Visit http://www.dovepress.com/ testimonials.php to read real quotes from published authors.

Submit your manuscript here: https://www.dovepress.com/clinical-interventions-in-aging-journal 